\title{
Three-Dimensional Endometrial Volume and Power Doppler Angiography for Prediction of Endometrial Carcinoma in Woman with Postmenopausal Bleeding
} Esmael Mohammed Talaat Elgarhy ${ }^{1}$, Mohammed Abd El-Kreem Kassem ${ }^{2}$, Mahmoud Abd El-Ghaffar Morsi Alzayat ${ }^{1}$

Obstetrics and Gynecology Department, ${ }^{1}$ Faculty of Medicine, Al-Azhar University, ${ }^{2}$ Medical Military Academy

\begin{abstract}
Background: A woman is considered menopausal after cessation of menstruation for one year. The goal of evaluations of postmenopausal bleeding (PMB) is to achieve the diagnosis with greatest accuracy, the least risk and expense for the patient. Transvaginal ultrasound (TVS) is imaging technique of choice for first line investigation of endometrial abnormalities as a possible cause of abnormal uterine bleeding, malignant and benign endometrial patterns can often be determined by TVS which can help diagnosis.

Objectives: This study aims to assess the accuracy of endometrial volume and vascularization index assessed by three-dimensional ultrasound in prediction of endometrial carcinoma in woman with postmenopausal bleeding.

Patients and Methods: This study was conducted in Ghamra Military Hospital on 100 women with postmenopausal bleeding.

Results: Our results revealed that both endometrial thickness, volume, and 3D-PDA indices may discriminate between endometrial cancer and benign conditions in women with postmenopausal bleeding. All parameters of our study were not significant in diagnosis of cancer in women with postmenopausal bleeding.

Conclusion: The diagnostic performance of the measurement of the size of the endometrium by three-dimensional ultrasound with respect to the distinction between benign and malignant endometriosis was higher when compared to the measurement of the thickness of the endometrium with 2D ultrasound.The Doppler flow indicators have three-dimensional power as good diagnostic tools in predicting endometrial cancer But it cannot rule out the presence of malignant tumors endometriosis.
\end{abstract}

Keywords: Three-Dimensional Endometrial Volume, Power Doppler Angiography, Endometrial Carcinoma, Postmenopausal Bleeding.

\section{INTRODUCTION}

A woman is considered menopausal after cessation of menstruation for one year ${ }^{(\mathbf{1})}$. The goal of evaluations of PMB is to achieve the diagnosis with greatest accuracy, the least risk and expense for the patient. Transvaginal ultrasound (TVS) is imaging technique of choice for first line investigation of endometrial abnormalities as a possible cause of abnormal uterine bleeding (2), malignant and benign endometrial patterns can often be determined by TVS which can help diagnosis ${ }^{(3)}$.

Current three dimensional ultrasound (3D U/S) technology allows storage of complete volumes and has capacity for routine clinical applications. All objects stored in these volumes can be represented in the multiplanar mode (coronal, transverse, sagittal) as well as in form of surface images.

The major advantage of multiplanar mode is that it enables simultaneous visualization of all three mutually perpendicular sectional planes on display screen ${ }^{(4)}$. Many physicians prefer to start with EMB, because it provides tissue samples for a histological diagnosis. It is easily performed, and causes minimal cramping. The test does have limitations, such as difficulty in obtaining adequate tissue samples ${ }^{(5)}$.

Dilatation and curettage (D\&C) procedure itself, although highly diagnostic, yet it has many limitations, including the need for general anesthesia, being a blind procedure with possible complications, only curettes $60 \%$ of the endometrial cavity and

missing a pathology such as: polyps and submucous myomas ${ }^{(6)}$.

With the advent of hysteroscopy in the last two decades, focus has shifted from endometrial biopsy to hysteroscopic-guided biopsy as a "gold standard" diagnostic tool in the evaluation of postmenopausal bleeding ${ }^{(7)}$.

Hysteroscopy permits direct visualization of cervical canal and uterine cavity. Diagnostic hysteroscopy is both accurate and feasible in diagnosis of intrauterine abnormalities. As diagnostic hysteroscopy predominantly performed in the outpatient clinic, and therapy in an inpatient setting, an accurate diagnosis is important to direct treatment at the specific pathology and avoid needless surgery. Moreover, "it may contribute to prognosis of expected quality of life" (8), but the diagnostic accuracy for hysteroscopy is high for endometrial cancer, polyps and submucous myomas but only moderate for endometrial hyperplasia ${ }^{(9)}$.

\section{AIM OF THE WORK}

This study aims to assess the accuracy of endometrial volume and vascularization index assessed by three-dimensional ultrasound in 
prediction of endometrial carcinoma in woman with postmenopausal bleeding.

\section{PATIENTS AND METHODS}

Study design: Prospective observational study

Setting: Ghamra Military Hospital.

Duration: From May 2018 to February 2019.

\section{Method of Protocol Ethics: \\ Ethical approval:}

The study protocol was approved for the research by the Ethical Committee, Faculty of Medicine, Al-Azhar University.

Patient information: The details of the procedure, aim of the work, benefit and risk of the trial had been explained to all patients.

Patient Consent: All participants signed informed consent (written consent) after explaining benefits and risks of the trial and have the right to leave the study at any time.

\section{Population:}

Patients with postmenopausal bleeding were recruited from attendants of the outpatient Gynecological Clinic and inpatient Gynecological ward at Ghamra Military Hospital.

The study was conducted on 100 cases of post-menopause women at age of 45-50 years old who passed one year after the last menstrual cycle, with body mass index $25-35 \mathrm{~kg} / \mathrm{m}^{2}$ with no family history of endometrial cancer, not on hormonal replacement therapy and they had not premenopausal history of irregular vaginal bleeding or any uterine pathology as fibroids.

\section{Methods}

\section{Inclusion criteria:}

1. Menopausal woman with natural menopause, which is defined as absence of menstruation for 1 year in (45-50) years old women ${ }^{(1)}$.

2. Patients complaining of postmenopausal bleeding.

\section{Exclusion criteria:}

1. Patients with bleeding tendency.

2. Patient with chronic diseases as (DM, HTN,... etc).

3. Patient taking anticoagulant drugs as warfarin or heparin.

4. Patients using tamoxifen citrate or any kind of hormonal replacement therapy.

5. Patient with history of previous diseases as (fibroid uterus or endometriosis).

6. Patient with previous history of PCO or other causes of infertility.

7. Nullipara patients

All patients were subjected to the following:
A- Detailed History: including the following points.

- Present history of bleeding including onset, course, duration, and criteria of bleeding pattern.

- History of recent hormonal contraception and particular drug intake.

- History of bleeding tendency or general cause of bleeding.

- Past history of operations or blood transfusion.

- Family history of similar condition.

- Menstrual history of menarche, last menstrual history, amount of bleeding.

\section{B- Examination}

I- General examination.

II- Local examination

\section{C- Investigations.}

Laboratory:

- Complete blood count.

- Coagulation profile.

- Fasting blood sugar.

- Liver and kidney functions.

\section{Radiology}

- Transvaginal ultrasound was done to measure endometrial thickness (>3 mm considered increased endometrial thickness) and/or detect any endometrial pathology.

- 3D power Doppler was done to reveal endometrial volume, endometrial thickness, uterine blood flow indices (FI, VI and VFI).

- Fractional curettage, biopsy sampling for pathological examinations were done also and better to be hysteroscopic guided.

- It was done using a Voluson E-6 machine (GE health care USA) with multifrequency transabdominal and transvaginal volumetric probes.

- All cases were performed by the same sonographer.

\section{Pathology}

- Fractional curettage and biopsy sampling for pathological examinations.

The cases were classified into two main groups:

A- Cases with normal endometrial thickness, volume and vascular indices which were categorized by biopsy sampling into:

AI - no pathological evidence

AII- With pathological endometrium which was subcategorized in to:

AIIa- Cases with premalignant lesions

AIIb- Cases with malignancy

B- Cases with abnormal endometrial thickness or volume or vascular indices or combined which were subcategorized by biopsy sampling into: 
BI- no pathological evidence

BII- With pathological endometrium which was subcategorized into:

BIIa-Cases with premalignant lesions

BIIb- Cases with malignancy.

\section{Statistical analysis}

Recorded data were analyzed using the statistical package for social sciences, version 20.0 (SPSS Inc., Chicago, Illinois, USA). Quantitative data were expressed as mean \pm standard deviation (SD). Qualitative data were expressed as frequency and percentage.

\section{The following tests were done:}

- Independent-samples t-test of significance was used when comparing between two means.
- Chi-square $\left(\mathrm{x}^{2}\right)$ test of significance was used in order to compare proportions between two qualitative parameters.

- The confidence interval was set to $95 \%$ and the margin of error accepted was set to $5 \%$. The pvalue was considered significant as the following:

- Probability (P-value)

- P-value $<0.05$ was considered significant.

- P-value <0.001 was considered as highly significant.

- P-value >0.05 was considered insignificant.

\section{RESULTS}

Table (1): Comparing endometrial thickness in different histopathological findings

\begin{tabular}{|l|c|c|}
\hline \multicolumn{1}{|c|}{ Histopathological result } & \multicolumn{2}{c|}{ Endometrial thickness (mm) } \\
\cline { 2 - 3 } \multicolumn{1}{|c|}{} & Mean \pm SD & Range \\
\hline Atrophic endometrium & $4.89 \pm 2.52$ & $1.2-9.6$ \\
Hyperplastic polyp & $5.64 \pm 3.46$ & $1.8-10$ \\
Simple endometrial hyperplasia without atypia & $4.83 \pm 2.61$ & $1.4-9$ \\
Complex endometrial hyperplasia & $6.13 \pm 3.59$ & $1.7-10$ \\
Simple endometrial hyperplasia with atypia & $6.73 \pm 4.11$ & $2.3-13.5$ \\
Adenocarcinoma & $6.63 \pm 3.15$ & $1.2-15$ \\
\hline P-value & \multicolumn{2}{|c|}{$\mathbf{0 . 1 8 5}$} \\
\hline
\end{tabular}
thickness

On comparing the different histopathological findings, there was insignificant difference in the endometrial

Table (2): Comparing endometrial volume in different histopathological findings

\begin{tabular}{|l|c|c|}
\hline \multicolumn{1}{|c|}{ Histopathological result } & \multicolumn{2}{c|}{ Endometrial volume $\left(\mathbf{c m}^{\mathbf{3}}\right)$} \\
\cline { 2 - 3 } & Median (IQR) & Range \\
\hline Atrophic endometrium & $7.9(3.9-13.2)$ & $1.8-45$ \\
Hyperplastic polyp & $3.7(2.9-15)$ & $1.9-20$ \\
Simple endometrial hyperplasia without atypia & $6.7(3.5-9.6)$ & $1.5-13.9$ \\
Complex endometrial hyperplasia & $28.9(15.1-37.5)$ & $2.4-45$ \\
Simple endometrial hyperplasia with atypia & $7(2.4-14)$ & $1.7-23.7$ \\
Adenocarcinoma & $13.9(7.1-15.8)$ & $1.2-23$ \\
\hline P-value & \multicolumn{2}{|c|}{$\mathbf{0 . 1 0 0}$} \\
\hline
\end{tabular}

On comparing the different histopathological findings, there was insignificant difference in the endometrial volume.

Table (3): Comparing VI values in different histopathological findings

\begin{tabular}{|l|c|c|}
\hline \multicolumn{1}{|c|}{ Histopathological result } & \multicolumn{2}{c|}{ VI } \\
\cline { 2 - 3 } & Median (IQR) & Range \\
\hline Atrophic endometrium & $2.35(1.9-9.1)$ & $0.07-38.9$ \\
Hyperplastic polyp & $10.32(2.4-13.5)$ & $2-15.7$ \\
Simple endometrial hyperplasia without atypia & $3.9(2.1-10.5)$ & $0.34-25.9$ \\
Complex endometrial hyperplasia & $8.85(5.05-14.4)$ & $2.5-18.7$ \\
Simple endometrial hyperplasia with atypia & $8.5(2.2-16)$ & $1.8-19.5$ \\
Adenocarcinoma & $9.45(2.8-19.8)$ & $1.2-47.7$ \\
\hline P-value & \multicolumn{2}{|c|}{$\mathbf{0 . 1 1 8}$} \\
\hline
\end{tabular}

On comparing the different histopathological findings, there was insignificant difference in the VI. 
Table (4): Comparing FI values in different histopathological findings

\begin{tabular}{|l|c|c|}
\hline \multicolumn{1}{|c|}{ Histopathological result } & \multicolumn{2}{c|}{ FI } \\
\cline { 2 - 3 } & Mean \pm SD & Range \\
\hline Atrophic endometrium & $19.48 \pm 9.25$ & $3-39$ \\
Hyperplastic polyp & $26.35 \pm 12.44$ & $17-55.01$ \\
Simple endometrial hyperplasia without atypia & $18.59 \pm 8.50$ & $10.1-32.5$ \\
Complex endometrial hyperplasia & $25.30 \pm 15.02$ & $9.1-39.1$ \\
Simple endometrial hyperplasia with atypia & $24.90 \pm 7.96$ & $15-35.2$ \\
Adenocarcinoma & $23.95 \pm 8.82$ & $11.1-39.5$ \\
\hline P-value & \multicolumn{2}{|c|}{$\mathbf{0 . 1 3 0}$} \\
\hline
\end{tabular}

$\bullet$ One Way ANOVA test

On comparing the different histopathological findings, there was insignificant difference in the FI.

Table (5): Comparing VFI values in different histopathological findings

\begin{tabular}{|l|c|c|}
\hline \multicolumn{1}{|c|}{ Histopathological result } & \multicolumn{2}{c|}{ VFI } \\
\cline { 2 - 3 } \multicolumn{1}{|c|}{ Redian (IQR) } & Range \\
\hline Atrophic endometrium & $1.8(0.6-9.8)$ & $0.01-22.03$ \\
Hyperplastic polyp & $3.7(0.7-7.3)$ & $0.3-20.8$ \\
Simple endometrial hyperplasia without atypia & $1.8(0.6-12.1)$ & $0.25-17.8$ \\
Complex endometrial hyperplasia & $6.15(2.8-11.7)$ & $0.5-16.2$ \\
Simple endometrial hyperplasia with atypia & $6.15(0.7-9.2)$ & $0.6-9.8$ \\
Adenocarcinoma & $10.97(5.1-15.1)$ & $0.12-20.8$ \\
\hline P-value & \multicolumn{2}{|c|}{$\mathbf{0 . 3 2 2}$} \\
\hline
\end{tabular}

On comparing the different histopathological findings, there was insignificant difference in the VFI.

Table (6): Comparing between benign and malignant groups in all parameters measured

\begin{tabular}{|c|c|c|c|c|}
\hline & & Benign & Malignant & P-value \\
\hline Endometrial thickness (mm) & $\begin{array}{l}\text { Mean } \pm \text { SD } \\
\text { Range }\end{array}$ & $\begin{array}{c}5.45 \pm 2.92 \\
1.2-15\end{array}$ & $\begin{array}{l}7.30 \pm 3.38 \\
1.7-15\end{array}$ & 0.064 \\
\hline Endometrial volume $\left(\mathrm{cm}^{3}\right)$ & $\begin{array}{l}\text { Median (IQR) } \\
\text { Range }\end{array}$ & $\begin{array}{c}7.95(3.5-14.9) \\
1.2-45\end{array}$ & $\begin{array}{c}14.05(10-15.8) \\
1.8-17.6\end{array}$ & 0.115 \\
\hline VI & $\begin{array}{l}\text { Median (IQR) } \\
\text { Range }\end{array}$ & $\begin{array}{c}5.06(2.1-11.5) \\
0.07-38.9\end{array}$ & $\begin{array}{c}10.75(2-21.3) \\
1.2-47.7\end{array}$ & 0.258 \\
\hline FI & $\begin{array}{l}\text { Mean } \pm \text { SD } \\
\text { Range }\end{array}$ & $\begin{array}{c}21.40 \pm 9.68 \\
3-55.01\end{array}$ & $\begin{array}{c}25.85 \pm 9.02 \\
11.1-39.5\end{array}$ & 0.168 \\
\hline VFI & $\begin{array}{l}\text { Median (IQR) } \\
\text { Range }\end{array}$ & $\begin{array}{c}4.75(0.6-11.9) \\
0.01-22.03\end{array}$ & $\begin{array}{c}9.6(5.1-18.7) \\
0.9-19.10\end{array}$ & 0.059 \\
\hline
\end{tabular}

\section{DISCUSSION}

This study was conducted in Ghamra Military Hospital on 100 women with postmenopausal bleeding. Our results revealed that both endometrial thickness, volume, and 3D-PDA indices may discriminate between endometrial cancer and benign conditions in women with postmenopausal bleeding. Several previous studies were published also around this study. Analyzing the diagnostic value of 3D-PDA indices in patients with postmenopausal bleeding for diagnosing endometrial cancer found that 3D-PDA indices was superior to endometrial thickness measurement for the detection of endometrial cancer in 100 women with postmenopausal bleeding.

Mansour et al. (10) reached the same conclusions by comparing endometrial volume and thickness in a series of 170 women with postmenopausal bleeding.

In our study all our parameters (endometrial thickness, endometrial volume, VI, FI, VFI) are not significant in diagnosis of the endometrial cancer in women with postmenopausal bleeding; as the p-value was $>0.05$.

Disagree with our study, a study was done by Gruboeck $\boldsymbol{e t} \boldsymbol{a l} .{ }^{(11)}$ analyzed the diagnostic value of endometrial volume in patients with postmenopausal bleeding for diagnosing endometrial cancer in a series of 97 women with postmenopausal bleeding. Endometrial thickness and volume in patients with normal or atrophic, hyperplasia or polyps was measured. In patients with endometrial cancer the endometrial thickness was $29.5 \mathrm{~mm}$ (SD 12.59) and the mean volume was $39.0 \mathrm{ml}$ (SD 34.16). The endometrial volume and thickness were significantly 
lower in patient with benign lesions. Thickness were the same in polyps (mean 15.3 \pm SD 5.21) and hyperplasia (mean16.0 \pm SD 5.64), however endometrial volume in hyperplasia (mean $8.0 \mathrm{ml} \pm$ SD 7.81) were significantly higher than ployp (mean $2.4 \pm$ SD 1.86). In 71 with normal or atrophic endometrium mean thickness and volumes were 5.3 $\mathrm{mm} \pm \mathrm{SD} 3.98$ and. $09 \mathrm{ml} \pm \mathrm{SD} 1.7$, respectively. This was less than abnormal cases. Endometrial thickness less than $5 \mathrm{~mm}$ was always associated with normal or atrophic endometrium. However there was overlap between normal cases and those with benign or malignant lesions. The best cut-off level for diagnosis of carcinoma was $15.0 \mathrm{ml}$ with sensitivity of 83.3 and specificity 82.2 , and positive predictive value of 54.4. The endometrial volume was higher in patients with carcinoma than those of benign lesion with cut-off level $13 \mathrm{ml}$ which mad sensitivity $100 \%$.

So, endometrial volume was superior to endometrial thickness measurement for the detection of endometrial cancer ${ }^{(\mathbf{1 1})}$.

Disagree to our study, Odeh et al. (12) included in their study not only postmenopausal women but also perimenopausal women (56 women with postmenopausal and 89 with perimenopausal bleeding were enrolled). The endometrial volume was $6.87 \mathrm{cc}$ in the normal histology group and 13.79 $\mathrm{cc}$ in the pathologic group in both menopausal and perimenopausal women (p 0.001 using MannWhitney test). Endometrial polyp did not affect the mean endometrial volume. The average volume of the endometrium in patients with carcinoma was 18.1 $\mathrm{cc}$ and $11.2 \mathrm{cc}$ in patients with hyperplasia; both were significantly higher than in the normal histology group

Disagree to our study, Mansour et al. ${ }^{(10)}$ on comparing endometrial volume and thickness in a series of 170 women with postmenopausal bleeding (patients with "endometrial atypia" was included in the group of endometrial cancer). In this study they measured endometrial thickness in the sagittal view of the uterus, including the anterior and posterior walls of endometrium. Endometrial volume was measured by VOCAL using the coronal plane of uterus, 1-mm shell thickness, and rotation steps of $30^{\circ}$. By the receiver operating characteristic curve, a higher sensitivity was found using endometrial volume than endometrial thickness for predicting atypia or malignancy. There were no statistical differences between the study and control groups.

Disagree with our study, Yamen et al. ${ }^{(13)}$ while studying 213 cases of postmenopausal bleeding they found that 42 cases (19.7\%) were cancer, 109 cases $(51.17 \%)$ were benign lesions (polyphyperplasia), 62 cases (29.1\%) were atrophic. They authenticated that both endometrial volume and thickness measurements by $3 \mathrm{D}$ and $2 \mathrm{D}$ scanning, respectively, were adequately reproducible but that the reproducibility of 3D was superior.

Agree with our study, Kim et al. ${ }^{(14)}$ playing an important role in discriminating endometrial cancer from benign diseases, which confirms the findings of previous published data regarding the correlation between endometrial cancer and endometrial volume. The positive cutoff value was offered for the prediction of malignancy with an endometrial thickness of 4.05 but the difference in this study that the women with a thin endometrium $(<5 \mathrm{~mm})$ were not excluded. The positive cutoff value was offered for the prediction of malignancy with an endometrial thickness of $9.5 \mathrm{~mm}$, not as the passive endometrial thickness of $5 \mathrm{~mm}$. This means that endometrial thickness of $>5 \mathrm{~mm}$ requires further evaluation with methods such as endometrial biopsy, catheter sampling, whole curettage, or hysteroscopic guided biopsy. However, an endometrium thicker than $9.5 \mathrm{~mm}$ is worthy of attention and is evaluated with malignancy in mind ${ }^{(14)}$.

Increased endometrial volume associated with a thick endometrium and, of course, can be related to endometrial cancer. It was assessed that the cutoff values of endometrial volume for the prediction of cancer was $4.05 \mathrm{~mL}$, which is not small compared with that of the reproductive endometrium (14).

On analyzing the receiver operating characteristic curves of endometrial for VI, FI, and VFI to assess their predictive value for malignancy; the area under the curve was statistically significant in all parameters for the prediction of endometrial cancer. VI, FI, and VFI have predictive power for malignancy before the performance of invasive endometrial biopsy. The best predictive cutoff values of the vascular parameters were relative values, but, in this mode setting, the cutoff values were 13.07 for VI, 12.61 for FI, and 3.76 for VFI. The study also revealed the predictive property of ultrasonography characteristics in comparison between endometrial hyperplasia and benign lesion the area under the curve for endometrial volume, VI and VFI were not significant (14).

Agree with our study, El-Mekkawi et al. ${ }^{(15)}$ reached the following result in sixty women with postmenopausal bleeding; 32 (53.3\%) women had benign endometrial lesions and $28(46.7 \%)$ had endometrial malignancy.

Women with malignancy tended to have significantly thicker endometrium (11.11 \pm 3.61 vs. $5.78 \pm 0.94 \mathrm{~mm})$, larger endometrial volume (4.89 \pm 3.53 vs. $2.22 \pm 1.6$ ), and higher 3DPD flow indices than those with benign endometrial lesions. There was a statistically significant difference between 3DPD and 3D multislice view, with increased vasculature in different planes in malignant cases than in benign cases. The best logistic 
regression models for predicting malignancy [i.e. the models with the largest area under the curve (AUC)] included endometrial thickness and VFI (AUC 0.984 and 0.884). Even though histopathological examination of the endometrium is the gold standard for the final diagnosis or exclusion of endometrial malignancy. Endometrial volume, 3DPD indices, and 3D multislice view are good diagnostic tools in predicting endometrial malignancy in women with postmenopausal bleeding ${ }^{(15)}$.

Hanafi et al. (16) didn't agree with our study as they stated that the ultimate discriminator between non-cancerous and cancerous endometrium was FI with a sensitivity of $85.7 \%$ and specificity of $98.2 \%$.

Disagree with our study, Opolskiene $\boldsymbol{e t}$ al. (17) found that the AUC of endometrial thickness was 0.82 , while that of endometrial volume was 0.78 . Of the 62 women included in the study; 49 (79\%) had benign endometrium and $13(21 \%)$ had malignant endometrium. The endometrial volume was larger and the flow indices were higher in both the endometrium and in the subendometrium in patients with malignant endometrium than in those with benign endometrium, but there was substantial overlap between the two categories. The best variables for discriminating between benign and malignant endometrium were endometrial thickness, and endometrial VI and VFI, all having AUC of 0.82. The best logistic regression model for predicting malignancy was that including the variables endometrial thickness and VI in the subendometrium with AUC of 0.86. Using the mathematically optimal risk cutoff value (0.22), the model correctly classified seven more benign cases, but two fewer malignant cases, than the best endometrial thickness cutoff $(11.8 \mathrm{~mm})$. Models including endometrial volume and flow indices performed less well than endometrial thickness alone (AUC 0.79 vs. 0.82). Intra-observer reliability was very high for all ultrasound variables. Power Doppler variables (VI and VFI in the endometrium) were not better than endometrial thickness.

Agree with our study, Odeh et al. (12) assessed the potential to differentiate malignancy and hyperplastic tissue from other non-cancerous conditions. Their study observed that 3-dimensional VIs are higher in endometrial cancer. The VI was not significantly higher in the hyperplasia group as expected, compared to patients with normal histology (2.03 and 2.27 respectively) while it was significantly higher in the carcinoma group compared to the normal group (4.45 and 2.27). The VI was 2.27 in the normal histology group and 2.95 in the pathologic group. The FI was 18.6 in the normal histology group and 23.8 in the pathologic group. The FI was significantly higher in the hyperplasia group compared to patients with normal histology (23.5 and
18.6 respectively), but was not significantly higher when compared to carcinoma alone (24.3 and 18.6). The VFI was 0.68 in the normal histology group and 0.89 in the pathologic group. The VFI was not significantly higher when comparing patients with normal histology and hyperplasia (0.68 and 0.58).

In disagreement with our study, in the study of Alcazar et al. ${ }^{(18)}$ power Doppler signals were identified in 84 of 91 cases $(92 \%)$. They were unable to identify power Doppler signals in 3 cases of endometrial polyps, 2 cases of endometrial hyperplasia, and 2 cases of cystic atrophy. However, they did not exclude these cases from analysis. All cases of endometrial cancer exhibited power Doppler signals. Mean endometrial volume, VI, and VFI were significantly higher in endometrial cancer, compared with all other benign diseases. FI was significantly higher in endometrial cancer, compared with endometrial polyp and hyperplasia but not when compared with cystic atrophy and submucous myomas. ROC curves showed that the best predictor for endometrial cancer was VI with an AUC of 0.90, which is significantly higher than all other parameters. However, they showed that 3dimensional VIs is not only higher in endometrial cancer, compared with endometrial hyperplasia, but also compared with other benign lesions (such as endometrial polyps or cystic atrophy). This indicates that endometrial vascularization is increased mainly in cases of adenocarcinoma.

So this analysis revealed that VI was the best parameter for the prediction of endometrial cancer with an AUC of 0.90, which is significantly higher than all other parameters ${ }^{(\mathbf{1 8})}$.

\section{CONCLUSION}

The diagnostic performance of the measurement of the size of the endometrium by three-dimensional ultrasound with respect to the distinction between benign and malignant endometriosis was higher when compared to the measurement of the thickness of the endometrium with 2D ultrasound.The Doppler flow indicators have three-dimensional power as good diagnostic tools in predicting endometrial cancer But it cannot rule out the presence of malignant tumors endometriosis.

\section{REFERENCES}

1. Goodman A (2014): Postmenopausal uterine bleeding. UpToDate. available at: https://www.uptodate.com/contents/postmenopau sal-uterine-bleeding

2. Leone FP, Timmerman D, Bourne $\mathrm{T}$ et al. (2010): Terms, definitions and measurements to describe the sonographic features of the endometrium and intrauterine lesions: a consensus opinion from the International Endometrial Tumor Analysis (IETA) group. Ultrasound Obstet Gynecol., 35(1):103-112. 
3. Dueholm M, Marinovskij E, Hansen ES et al. (2015): Diagnostic methods for fast-track identification of endometrial cancer in women with postmenopausal bleeding and endometrial thickness greater than $5 \mathrm{~mm}$. Menopause, 22(6):616-26.

4. Merze E, Benott B, Blaas HG et al. (2007): Standardization of three dimensional images in obstetrics and gynecology: consensus statement. Ultrasound Obstet Gynecol., 29: 697-703.

5. Hosoi A, Ueda Y, Shindo M (2013): Endometrial thickness measured by ultrasonography in postmenopausal patients with endometrial carcinoma has significance, irrespective of histological subtype. Int J Gynecol Cancer, 23(7):1266-1269.

6. Mencaglia L, Hamou JE (2000): Manual of Gynecologic Hysteroscopy: diagnosis and surgery. Endopress Tuttlingen, 24: 63-66.

7. Ribeiro CT, Silva JC, Candio Dos Reis FJ et al. (2007): Hysteroscopy as standard procedure for assessing endometrial lesions among postmenopausal women. Saso Palou Med J., 125:338-342.

8. Van Dongen H, de Kroon CD, Jacobi CE et al. (2007): Diagnostic hysteroscopy in abnormal uterine bleeding: a systematic review and metaanalysis. BJOG., 114(6):664-675.

9. Gkrozou F, Dimakopoulos G, Vrekoussis T et al. (2015): Hysteroscopy in women with abnormal uterine bleeding: a meta-analysis on four major endometrial pathologies. Arch Gynecol Obstet., 291(6):1347-54.

10. Mansour GM, El-Lamie IK, El-Kady MA et al. (2007): Endometrial volume as predictor of malignancy in women with postmenopausal bleeding. Int J Gynaecol Obstet., 99:206-10.

11. Gruboeck K, Jurkovic D, Lawton F et al. (1996): The diagnostic value of endometrial thickness and volume measurements by three dimensional ultrasound in patients with menopausal bleeding. Ultrasound Obstet Gynecol., 8: 272-6.

12. Odeh M, Vainerovsky I, Grinin V et al. (2007): Three- dimensional endometrial volume and 3dimensional power Doppler analysis in predicting endometrial carcinoma and hyperplasia. Gynecol Oncol., 106: 348-53.

13. Yaman $\mathrm{C}$, Habelsberger $\mathrm{A}$, Tews $\mathrm{G}$ et al. (2008): The role of three-dimensional volume measurement in diagnosing endometrial cancer in patients with postmenopausal bleeding. Gynecologic Oncology, 110(3):390-5.

14. Kim A, Lee JY, Chun S et al. (2015): Diagnostic utility of three-dimensional power Doppler ultrasound for postmenopausal bleeding Taiwanese Journal of Obstetrics and Gynecology, 54(3):221-226.

15. El-Mekkawi SFA, Bahaa AMA, Akl SAA et al. (2015): Three-dimensional power Doppler ultrasound with a three-dimensional multislice view: is it a new modality in endometrial evaluation in women with postmenopausal bleeding? Evidence Based Women's Health Journal, 5 (3): 143-149.

16. Hanafi S, Abou-gabal A, Akl S et al. (2014): Value of three dimensional power Doppler ultrasound in prediction of endometrial carcinoma in patients with postmenopausal bleeding. J Turk Ger Gynecol Assoc., 15: 78-81.

17. Opolskiene G, Sladkevicius $P$, Jokubkiene $L$ et al. (2010): Three-dimensional ultrasound imaging for discrimination between benign and malignant endometrium in women with postmenopausal bleeding and Sonographic endometrial thickness of at least 4.5 mm. Ultrasound Obstet Gynecol., 35:94-102.

18. Alcazar JL, Castillo G, Minguez JA et al. (2003): Endometrial blood flow mapping using transvaginal power Doppler sonography in women with postmenopausal bleeding and thickened endometrium. Ultrasound Obstet Gynecol., 